\title{
The light elements in a helio- and asteroseismic perspective
}

\author{
Sylvie Vauclair ${ }^{1}$ \\ ${ }^{1}$ Laboratoire d'Astronomie de Toulouse-Tarbes, Université de Toulouse, \\ 14 Avenue Edouard Belin, 31400 Toulouse, France \\ email: sylvie.vauclair@ast.obs-mip.fr
}

\begin{abstract}
Asteroseismology is a powerful tool to derive stellar parameters, including the helium content and internal helium gradients, and the macroscopic motions which can lead to lithium, beryllium, and boron abundance variations. Precise determinations of these parameters need deep analyses for each individual stars. After a general introduction on helio and asteroseismology, I first discuss the solar case, the results which have been obtained in the past two decades, and the crisis induced by the new determination of the abundances of heavy elements. Then I discuss asteroseismology in relation with light element abundances, especially for the case of main sequence stars.
\end{abstract}

Keywords. Sun: abundances, heliosismology - stars: interiors

\section{Introduction}

The general study of stellar oscillations began long before the advent of the so-called helio and asteroseismology. In the past, astronomers only detected large amplitude oscillations and they spoke of "variable stars" or "pulsating stars". Nowadays, new techniques allow to detect very small amplitude oscillations, and variable stars are known all over the HR diagram. They can be classified according to:

- the type of waves which leads to their oscillations, either pressure of gravity waves or both

- their amplitudes

- their excitation mechanisms

Before the first discovery of solar oscillations, sola- type stars were not supposed to be variable, as the acoustic waves are damped in their interiors. We now know that stochastic excitation induced by convective motions leads to permanent destabilisation so that these stars behave like resonant cavities in spite of the waves damping.

The first report of a periodic solar velocity field was given by Leighton et al. (1962). Evidences of the five minute oscillations were later confirmed by Ulrich (1970) and Leibacher \& Stein (1971). Some 10 millions p-modes are observed in the Sun, with frequencies around 2 to $4 \mathrm{mHz}$, velocity amplitudes about one $\mathrm{cm} . \mathrm{s}^{-1}$ (max $20 \mathrm{~cm} . \mathrm{s}^{-1}$ ), relative variations of brilliance $10^{-7}$, mode lifetimes of a few hours up to a few months.

All the solar-type stars which have been observed for seismology do oscillate with frequencies in the interval 0.1 to $10 \mathrm{mHz}$. However, only global modes can be detected, as stellar surfaces cannot be resolved. A few tens of modes only can typically be identified in such stars, so that the inversion techniques and the precision on the results are quite different from the solar case.

In this framework, light elements are related to helio- and asteroseismology in various ways. Helium 4, the second most abundant element in stars, is the only one which 
directly influences the oscillation scheme, as modifying its abundance changes the stellar structure. The helium abundance and abundance gradients may be derived from seismic studies. It can also be the cause for seismic destabilisation of the star through $\kappa$-mechanism.

On the other hand, the light elements lithium, beryllium, boron, and helium 3 are only indirectly related to asteroseismology, as they are destroyed by nuclear reactions in a way determined by macroscopic motions (convective zones, overshooting, internal mixing) which themselves have seismic signatures. As for deuterium, I do not think that any relation can be found between its abundance evolution and helio or asteroseismology.

\section{The solar case}

Helioseismology consists in analysing the sound waves that propagate throughout the Sun and using them to measure, by inversion procedures, the solar internal parameters like temperature, pressure, density, helium abundance, partial ionisation regions, zones of convection and macroscopic motions, internal rotation.

The oscillation modes are trapped in spherical-shell cavities extending between the surface and their "turning points", which are consequences of the refraction of the waves due to the inwardly increasing sound velocity. The angular component of the wave function of these oscillations is described by the spherical harmonics, characterized by their two quantum numbers: the degree $l$ and the azimuthal-order $m$. The number $l$ corresponds to the number of null circles around the sphere, and $m$ to the number of these null circles which cross the poles (meridional circles). In the radial direction, the number of null spheres is characterized by the third quantum number $n$. The depth of a given cavity depends on both the oscillation frequency and the spherical-harmonic degree $l$ of the associated mode. Modes with large $l$ are confined near the surface, while modes with small $l$ extend much deeper, those with $l=0$ and $l=1$ reaching the center of the Sun itself. Consequently, all these modes sample different, although overlapping, regions of the solar interior.

Various observational techniques have been developed to detect and precisely observe the solar oscillation frequencies. To obtain the needed precision, the observations must go on uninterrupted during at least one month (a solar rotation), which may be reached by three different solutions: instruments at the Earth's poles (Antarctica), space observations (e.g. SOHO: sohowww.nascom.nasa.gov) and networks of instruments dispatched in longitude all around the Earth (e.g. GONG: gong.nso.edu).

Inversion procedures have been developed to obtain very precisely the sound velocity throughout the Sun, and the individual internal parameters by comparison with models. Owing to the very large number of observed resonant modes, and to their propagations in different internal cavities, at different depths, it is possible to derive the solar internal parameters with an accuracy of 0.1 percent in most of the internal Sun (see Basu et al. 2009 and references therein). The first important success of helioseismology was that the seismic profile of the sound velocity inside the Sun clearly indicated the exact depth of the convective zone, at a fractional radius $0.713+/-0.03$ (Christensen-Dalsgaard et al.1996). It also showed that overshooting was not present or extended on a very small depth if any.

In the early phases of seismic inversion and comparison with solar models, around 1995, discrepancies of order one percent were found. Then it was realized that helium diffusion, which decreases the helium abundance in the convective zone by about 20 percent compared to the internal one, had to be added to obtain better agreement. Other physical processes were improved, motivated by these new constraints: opacities 
and equations of state (e.g. OPAL, Iglesias \& Rogers 1996, Rogers \& Nayfonov 2002) and nuclear reaction rates (Angulo et al. 1999). It was also found that a mild turbulence below the convective zone, which could smoothen the diffusion-induced helium gradient, was necessary to obtain a good fit between the seismic and model sound velocity in this special region. This mild turbulence was able to account for the lithium deficiency observed in the solar outer layers, as well as the constraints imposed by beryllium (Balachandran \& Bell 1997) and helium 3 (Geiss \& Gloecker 1998)(see Richard et al. 1996, Brun et al. 2002).

Precise analysis of the rotational splitting of the solar mode frequencies allowed to discover that, while the outer solar layers undergo a well-known differential rotation, the internal Sun, below the convective zone, rotates as a solid body. This offered an important challenge to hydrodynamicists and is not entirely solved. It may be related to internal gravity waves (Charbonnel \& Talon 2005, Talon \& Charbonnel 2008), or to the internal solar magnetic field (Turck-Chieze et al. 2005).

The picture of our Sun seemed to have well improved during about 20 years, with very good results, until a crisis came with the new determinations of the solar chemical composition by Asplund et al. (2005). Using 3D simulations of the atmospheric solar motions, they determined heavy element abundances significantly smaller than obtained before (Grevesse \& Sauval 1998). Although these abundances have recently been revised and slighty increased (Asplund et al. 2009), the discrepancy between the models computed with the new abundances and the seismic inversions is unacceptable. As the new determinations of abundances seem convincing, there is a real challenge about solar physics (see Serenelli et al. 2009).

Several ideas have emerged to try and solve this discrepancy. None of them worked. The most promising one may be the idea of accretion by the young Sun of metal-poor material coming from the planetary disc gas (Castro et al. 2007). However, this leads to a steep abundance gradient below the convective zone, incompatible with the present sound velocity. New studies are under work, to see how this steep gradient could be smoothed, without modifying too much the internal Sun. Another solution could be that a systematic error occurred in the determinations of the new abundances, but at the present time it does not seem to be the case.

\section{The stellar case}

Asteroseismology can give much more precise values of the stellar parameters, including age, mass, radius, stellar gravity, effective temperature, metallicity, helium abundance value, depth of convective regions, than any other means. Such precise determinations need deep seismic analyses of individual stars, and cannot be obtained with approximate theories only, although these approximate theories are very useful for first insights.

The stellar oscillations may be observed either by spectroscopic observations, using Doppler effect on lines, or by photometric method, which gives access to the global luminosity variations of the star. For technical reasons, the first method is used on the ground (e.g. HARPS and SOPHIE spectrographs) while the second one is used on satellites, like COROT or KEPLER. In any case, only global modes can be detected, with $l$ values ranging from 0 to 3 . Typically a few tens of modes at most can be identified in stars, far from the ten millions observed in the Sun.

The inversion techniques used for the solar case cannot be applied to stars. In particular, the outer layers are not scanned by the different waves as it is in the Sun, as all the observed modes travel deep inside. However, if the waves encounter regions of rapid variation of the sound velocity on their way, they are partially reflected, creating 
secondary periods. This phenomenon is very helpful in the determination of internal stellar characteristics, as will be seen below.

Tassoul (1980) derived an asymptotic analytical expression for the mode frequencies in stellar cavities, which is widely used as a first approximation:

$$
\nu_{n, \ell} \simeq 2 \pi \Delta \nu_{0}\left(n+\frac{\ell}{2}+\epsilon\right)
$$

with

$$
\Delta \nu_{0}=\left[2 \int_{0}^{R} \frac{1}{r} d r\right]^{-1},
$$

which is the inverse of twice the time needed by the sound waves to travel from the stellar surface down to the center. This quantity is named the mean large separation.

One must be aware however that this theory is a crude approximation, valid only for small $l$ and large $n$ values, and only in stars in which the sound velocity varies smoothly as in the Sun. In a real star, there may be strong deviations from this theory and these deviations do provide important information about specific regions of the star (Soriano et al. 2007, see below).

In the first order approximation, two p-modes of successive radial order $n$, with the same degree $\ell$ are approximately separated by $\Delta \nu_{0}$. If we define the large separation by:

$$
\Delta \nu=\nu_{n+1, \ell}-\nu_{n, \ell}
$$

we have $\Delta \nu_{n, \ell}$ equal to $\Delta \nu_{0}$. But deviations from the asymptotic theory induce deviations of $\Delta \nu$ from $\Delta \nu_{0}$.

It is also useful to define the small separations as:

$$
\delta \nu=\nu_{n, \ell}-\nu_{n-1, \ell+2}
$$

At first order we clearly have $\delta \nu_{n, \ell} \simeq 0$. But in a real star, there are deviations from this theory and the small separations are not equal to zero. These quantities are very sensitive to the deep stellar interior (Gough 1986, Roxburgh 2009, and references therein) and can give us precious informations about the stellar core. These quantities, and in particular for the degrees $\ell=0-\ell=2$, can even become negative (Soriano et al. 2007, Soriano \& Vauclair 2008). This specific behaviour is related to the presence of a convective core or to a helium core with sharp edges. We can use this phenomenon to characterize helium-rich cores and to give strong constraints on the possible overshooting.

Finally, in regions with an important gradient of the sound velocity, like the boundary of the convective zone or the HeII ionization zone, there is partial reflexions of the waves that create modulations on the frequencies. These modulations clearly appear in the so-called second differences (Gough 1990, Vauclair \& Theado 2004) which are defined by:

$$
\delta_{2} \nu=\nu_{n+1, \ell}+\nu_{n-1, \ell}-2 \nu_{n, \ell}
$$

The modulation period of the oscillations is equal to twice the acoustic depth, which is the time needed for the sound waves to travel from the considered region to the stellar surface:

$$
t_{s}=\int_{r_{s}}^{R} \frac{d r}{c(r)}
$$

$c(R)$ is the sound velocity at the radius $r$, and $r_{s}$ the radius of the considered region. 


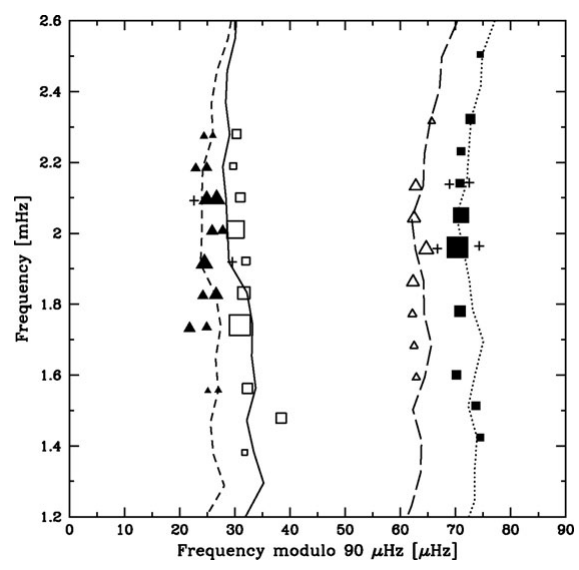

Figure 1. Example of echelle diagram: the star mu Arae. The symbols represent the observed modes, empty squares, $l=0$, filled squares, $l=1$, filled triangles, $l=2$ and empty triangles, $l=3$. The lines correspond to a stellar model. When two symbols are present, they correspond to two peaks due to rotational splitting.

A useful representation of the oscillation frequencies is the echelle diagram (Figure 1). In ordinates are plotted the frequencies and in abscissas, the same frequencies modulo the mean large separation. According to the asymptotic expressions, we should obtain straight lines corresponding to each value of the degree $\ell$. In real stars, the specific features observed in the echelle diagramme structure are indicative of their internal structure.

\section{Helium determinations from asteroseismology}

\subsection{Helium in stellar outer layers and helium gradients}

Rapid variations of the sound velocity in the outer stellar layers lead to partial reflections of the sound waves, which appear as frequency modulations in the "second differences". The periods of these modulations are equal to $2 t_{s}$ where $t_{s}$ is the time needed for the acoustic waves to travel between the surface and the considered region (acoustic depth).

These rapid variations can be due to the boundary of the outer convective zone, to helium ionisation regions and to diffusion-induced helium gradients. In practice, when helium settling is taken into account, the importance of helium gradients is larger than those of the convective boundary and helium ionisation regions (Vauclair \& Theado 2004, Castro \& Vauclair 2006).

Similar computations can be done for evolutionary models in which helium diffusion is introduced or suppressed. The resulting second differences are quite different. When diffusion is suppressed, they show signatures of the helium ionization zones while the signatures of helium gradients have, of course, disappeared.

Houdek \& Gough (2007) have shown in detail how to determine the helium abundance in stars from the observed helium-ionisation-induced features observed in the second differences. It gives interesting and precise results, but one must keep in mind that the obtained value corresponds to the helium abundance inside the convective zone, not the original one, as it has been decreased by gravitational settling.

\subsection{Determinations of the internal helium abundance from model comparisons}

Stars have to be observed during a sufficiently long time, typically eight nights or more with the HARPS spectrograph, several months for space observations, to allow precise 
comparisons with models. The Fourier analysis may lead to mode identification, as discussed in Bouchy et al. (2005), and frequency determinations. Meanwhile evolutionary tracks are computed, with various input parameters (mass, chemical composition, presence or not of overshooting, etc...). The computed mode frequencies are compared with the observed ones in a rigorous way. In this framework, departures from the "asymptotic theory" give fundamental information on the stellar parameters and internal structure.

For a given set of abundances (metallicity and helium value), only one model may reproduce the observed frequencies in a satisfying way (Soriano et al. 2007, Vauclair et al. 2008). The various models obtained in this way for different chemical composition have similar ages, gravities, and radii. The other parameters are then constrained with the help of the spectroscopic observational boxes.

In this way, it becomes possible to determine the internal helium abundance of the stars. Up to now, it has been done for two main-sequence exoplanet-host stars, which are both overmetallic compared to the Sun. The results for the helium abundance are quite different.

The exoplanet-host star $\mu$ Arae (HD160691) is a G5V star which has been observed for seismology during 8 nights in August 2004 with HARPS. The observations allowed to identify 43 oscillation modes of degrees $l=0$ to $l=3$ (Bouchy et al. 2005). They have been analysed by Bazot et al. (2005), and by Soriano et al. (2007) after revision of its Hipparcos parallax. The metallicity of this star, compared to the solar one, is $[\mathrm{Fe} / \mathrm{H}]=$ $0.30 \pm 0.01$, and the helium abundance is correspondingly high, $Y=0.301 \pm 0.01$.

Solar-type oscillations of the exoplanet-host star $\iota$ Hor (HD17051) were detected with HARPS in November 2006. Up to 25 oscillation modes could be identified and compared with stellar models. The analysis is discussed in detail in Laymand \& Vauclair (2007) and Vauclair et al. (2008). Contrary to $\mu$ Arae, which has a high helium value, as expected from the normal evolution of the chemical abundance of galaxies, according to its high metallicity, ८ Hor has a very small helium value, comparable to that of the Hyades: $Y=0.255 \pm 0.015$, for a metallicity $[\mathrm{Fe} / \mathrm{H}]=0.16 \pm 0.02$. As we also know that this star has the same kinematics as the cluster in the Galaxy, this is an indication that it was formed with the cluster and evaporated.

\section{Helium abundance variations and kappa mechanism}

Helium is an important element in the framework of oscillation stars, as it may in some cases lead to wave amplification though kappa mechanism. Here I only discuss the Am $\delta$-Scuti case.

Among the main sequence stars which lie inside the instability strip, many chemically peculiar stars are found. The so-called Am stars are found in the H.R. diagram at the same place as the $\delta$-Scuti stars. Generally speaking, the former ones show abundance peculiarities, namely a general overabundance of metals (except calcium and scandium), but no oscillations, while the later ones are pulsating but chemically normal. As discussed by Turcotte et al. (2000), in A-type stars, almost $70 \%$ of non chemically peculiar stars are $\delta$-Scuti variables at current levels of sensitivity while most non-variable stars are Am stars. Furthermore, Am stars are slower rotators than $\delta$-Scuti stars. In this region of the H.R. diagram, the stars display two different convective zones in their outer layers : the upper one due to the $\mathrm{HI}$ and HeI ionisations and the lower one to the HeII ionisation. The $\delta$-Scuti stars pulsate due to a $\kappa$-mechanism which takes place in the second convective zone. When microscopic diffusion occurs, this convective zone disappears due to helium depletion and the $\kappa$-mechanism cannot take place anymore (Vauclair et al. 1974). 
Some oscillating Am stars have been discovered (Kurtz 1989), which challenge the previously accepted theory. Richer et al. (2000) and Turcotte et al. (2000) computed models of Am stars in the framework of the Montreal models. They found that, due to the iron accumulation in the radiative zone below the $\mathrm{H}$ and He convective zone, a new convective region appears which increases the diffusion time scales compared to the previous models. In these new models, helium is still substantially present in the helium convective zone at the ages of the considered stars. They claim that it is possible to account for the existence of oscillating Am stars close to the cool boundary of the instability strip.

These computations ignored however an important process which occurs in case of $\mu$-gradient inversion : thermohaline convection, or double-diffusive convection (Vauclair 2004, Charbonnel \& Zahn 2007). Recently, Theado et al. (2009) have done precise computations of such diffusion-induced accumulation of elements, including thermohaline convection. The accumulation is much smaller than previously thought, but still present. This study will lead to many applications concerning element abundances in stars.

\section{Conclusion}

From the few examples already available, asteroseismology has proved to be a powerful tool for determining stellar parameters and constraints on their internal structure. However, tests have to be done for individual stars, observed during long periods. Usual approximate theories are not precise enough to obtain such results.

Only helium can be directly determined from asteroseismology. Lithium, beryllium, and boron, whose abundances are obtained from spectroscopy, are related to the macroscopic motions at work below the outer convective zones in solar-type stars. Asteroseismology can be used to constrain these macroscopic motions, in some cases. At least the depth of the convective zones can be derived somewhat precisely. Other kinds of mixing, like rotation-induced mixing, can influence the mode frequencies (e.g. Eggenberger 2009), but up to now the observed precision in the modes identifications and frequencies is not enough to measure it. Maybe in the future?

\section{References}

Angulo C., Arnould M., \& Rayet M., (NACRE collaboration) 1999, Nuclear Physics A, 656, 1 Asplund, M., Grevesse, N., \& Sauval, J. 2005, Cosmic Abundances as Records of Stellar Evolution and Nucleosynthesis, 336, 25

Asplund, M., Grevesse, N., Sauval, J., \& Scott, P. 2009, ARA\&A, 47, 481

Balachandran, S. \& Bell, R.A. 1997, AAS meeting 29, 1325

Basu, S., Chaplin, W. J., Elsworth, Y., New, R., \& Serenelli, A. M. 2009, ApJ, 699, 1403

Bazot, M., Vauclair, S., Bouchy, F., \& Santos, N. C. 2005, A\&A, 440, 615

Brun, A. S., Antia, H. M., Chitre, S. M., \& Zahn, J.-P. 2002, A\&SA, 391, 725

Bouchy, F., Bazot, M., Santos, N. C., Vauclair, S., \& Sosnowska, D. 2005, A\&A, 440, 609

Castro, M. \& Vauclair, S. 2006, A\&A, 456, 611

Castro, M., Vauclair, S., \& Richard, O. 2007, A\&A, 463, 755

Charbonnel, C. \& Talon, S. 2005, Science, 309, 2189

Charbonnel, C. \& Zahn, J. P. 2007, A\&A (Letters), 467, 15

Christensen-Dalsgaard J. et al. 1996, Science, 272, 1286

Eggenberger, P. 2009, this conference

Geiss, J. \& Gloecker, G. 1998, Space Science Reviews, 84, 239

Gough, D. O. 1986, in Hydrodynamic and magnetohydrodynamic problems in the Sun and stars, ed. Y. Osaki (Uni. of Tokyo Press), p. 117 
Gough, D. O. 1990, In Progress of Seismology of the Sun and Stars, Proc. Oji International Seminar (Hakone) (Japan : Springer Verlag), Lect. Notes Phys., 367, 283, eds Y. Osaki, H. Shibahashi

Grevesse, N. \& Sauval, A. J. 1998, Space Science Reviews, 85, 161

Houdek, G. \& Gough, D.O. 2007, MNRAS, 375, 861

Iglesias, C. A. \& Rogers, F. J. 1996, ApJ, 464, 943

Kurtz, D. W. 1989, MNRAS, 238, 1077

Laymand, M. \& Vauclair, S. 2007, A\&A, 463, 657

Leibacher, J. W. \& Stein, R. F. 1971, APL, 7, 191L

Leighton, R. B., Noyes, R. W., \& Simon, G. W. 1962, ApJ, 135, 474

Richard, O., Vauclair, S., Charbonnel, C., \& Dziembowski, W. A. 1996, A\&\&A, 312, 1000

Richer, J., Michaud, G., \& Turcotte, S. 2000, ApJ, 529, 338

Rogers, F. J. \& Nayfonov, A. 2002, ApJ, 576, 1064

Roxburgh, I. W 2009, A\&AA, 493, 185

Serenelli, A. M., Basu, S., Ferguson, J. W., \& Asplund, M. 2009, ApJ, 705, L123

Soriano, M., Vauclair, S., Vauclair, G., \& Laymand, M. 2007, A\&AA, 471, 885

Soriano, M. \& Vauclair, S. 2008, A\&A 488, 975

Talon, S. \& Charbonnel, C. 2008, A\&A, 482, 597

Tassoul, M. 1980, ApJS, 43, 469

Theado, S., Vauclair, S., Alecian, G. \& Le Blanc, F. 2009, ApJ, 704, 1262

Turck-Chieze, S., Appourchaux, T., Ballot, J., et al., 2005, ESASP, 588, 193

Turcotte, S., Richer, J., Michaud, G., \& Christensen-Dalsgaard, J. 2000, A\& $A, 360,603$

Ulrich, R. K. 1970, ApJ, 162, 993

Vauclair, G., Vauclair, S., \& Pamjatnikh, A. 1974, A\&A, 31, 63

Vauclair, S. 2004, ApJ, 605, 874

Vauclair, S. \& Théado, S. 2004, A\& A, 425, 179

Vauclair, S., Laymand, M., Bouchy, F., Vauclair, G., Hui Bon Hoa, A., Charpinet, \& S., Bazot, M. 2008, A\&A (Letters), 482, 5 